University of Nebraska - Lincoln

DigitalCommons@University of Nebraska - Lincoln

November 1990

\title{
Effects of Magnetic Field and Temperature on the Critical Current Hysteresis in TI-Ca-Ba-Cu-O Thin Films
}

\author{
Hung-Lun Chang \\ Institute of Electro-Optical Engineering, National Chiao Tung University \\ Jenh-Yih Juang \\ Institute of Electrophysics, National Chiao Tung University \\ Sy_Hwang Liou \\ University of Nebraska-Lincoln, sliou@unl.edu \\ Tzeng-Ming Uen \\ Institute of Electrophysics, National Chiao Tung University \\ Yih-Shun Gou \\ University of Nebraska - Lincoln
}

Follow this and additional works at: https://digitalcommons.unl.edu/physicsliou

Part of the Physics Commons

Chang, Hung-Lun; Juang, Jenh-Yih ; Liou, Sy_Hwang; Uen, Tzeng-Ming ; and Gou, Yih-Shun, "Effects of Magnetic Field and Temperature on the Critical Current Hysteresis in TI-Ca-Ba-Cu-O Thin Films" (1990). SiHwang Liou Publications. 48.

https://digitalcommons.unl.edu/physicsliou/48

This Article is brought to you for free and open access by the Research Papers in Physics and Astronomy at DigitalCommons@University of Nebraska - Lincoln. It has been accepted for inclusion in Si-Hwang Liou Publications by an authorized administrator of DigitalCommons@University of Nebraska - Lincoln. 


\title{
Effects of Magnetic Field and Temperature on the Critical Current Hysteresis in Tl-Ca-Ba-Cu-O Thin Films
}

\author{
Hung-Lun Chang, Jenh-Yih JuAnG ${ }^{\dagger}$, Sy-Hwang Liou ${ }^{\dagger \dagger}$, \\ Tzeng-Ming UEN ${ }^{\dagger}$ and Yih-Shun $\mathrm{GoU}^{\dagger}$ \\ Institute of Electro-Optical Engineering, National Chiao Tung University, \\ Hsinchu, Taiwan, 30050, R.O.C. \\ ${ }^{\dagger}$ Institute of Electrophysics, National Chiao Tung University, \\ Hsinchu, Taiwan, 30050, R.O.C. \\ ${ }^{+}$Behlen Laboratory of Physics, University of Nebraska-Lincoln, \\ Lincoln, NE 68588-0111, U.S.A.
}

(Received August 13, 1990; accepted for publication October 11, 1990)

\begin{abstract}
The behavior of the critical current density of $\mathrm{Tl}-\mathrm{Ca}-\mathrm{Ba}-\mathrm{Cu}-\mathrm{O}$ superconducting thin films in a cycling magnetic field was investigated. The hysteresis of the transport $J_{c}$ manifested similar features to those found in polycrystalline $\mathrm{Y}-\mathrm{Ba}-$ $\mathrm{Cu}-\mathrm{O}$ and granular $\mathrm{Nb}, \mathrm{Al}$ thin films in zero-field cooling. However, a novel result of the transport $J_{\mathrm{c}}$ in field-cooled samples has been observed. The results were attributed to the presence of a saturated trapped magnetic flux in the weaklink network. The effects of temperature on both the trapped flux and the transport $J_{\mathrm{c}}$ suggest the existence of various states of the trapped flux in the Tl-based superconducting thin films.
\end{abstract}

KEYWORDS: TI-Ca-Ba-Cu-O thin films, critical current, hysteresis, flux trapping

It is commonly accepted that the granular nature of all the high- $T_{\mathrm{c}}$ superconductor materials leads to their weaklink-dominated behavior in the intergranular current transfer and a small intergranular $H_{\mathrm{c} 2 \mathrm{j}} .{ }^{1)}$ As a result, the transport critical current density $\left(J_{\mathrm{c}}\right)$ of the high- $T_{\mathrm{c}}$ superconductors is markedly depressed even in very low applied magnetic fields and elicits a strong hysteresis when the field is cycled due to flux trapping. Recently, several groups have reported the observation of critical current irreversibility in polycrystalline $\mathrm{Y}-\mathrm{Ba}-\mathrm{Cu}-\mathrm{O}$ superconductors, ${ }^{2-5)}$ which shows a similar feature of transport $J_{c}$ in granular aluminum and niobium thin film bridges. ${ }^{6)}$ The field-dependent critical current density $\left(J_{c}(H)\right)$ was evidently enhanced while the magnetic field was reduced in its maximum value compared to its original increasing path. Such history-dependent behavior of $J_{\mathrm{c}}(H)$ is believed to be caused by the interactions between intergrain and intragrain supercurrents. ${ }^{2-5)}$ However, similar experiments in $\mathrm{Bi}-\mathrm{Sr}-\mathrm{Ca}-\mathrm{Cu}-\mathrm{O} / \mathrm{Ag}$ superconducting wires $^{7)}$ demonstrated the very weak or no hysteretic behavior of $J_{c}$, indicating that the history-dependent effect may not be universal and is sample-dependent. In this letter, systematic studies of temperature and low magnetic field $(<3000 \mathrm{Oe})$ dependences of $J_{\mathrm{c}}$ on a series of $\mathrm{Tl}-\mathrm{Ca}-\mathrm{Ba}-\mathrm{Cu}-\mathrm{O}$ superconducting thin films with vastly different microstructure and zero field superconducting properties are reported. The results confirmed that the history-ependent $J_{\mathrm{c}}(H)$ is indeed a sample-dependent property. Moreover, the equilibrium properties of flux lines as delineated by the temperature effects showed three distinct regimes which are believed to be responsible for the results observed.

Most of the Tl-Ca-Ba-Cu-O superconducting thin films used in the present study were made by single-target dc sputtering with post-annealing. ${ }^{8)}$ The films were 1 to $1.2 \mu \mathrm{m}$ thick. The film microstructure as delineated by scanning electron microscopy (SEM) shows a rather rough appearance with network-like permuted grains, although the $\mathrm{X}$-ray diffraction shows a mostly $c$-axisoriented structure. Critical current densities were measured by the standard four-probe technique with a 1 $\mu \mathrm{V}$ criterion in the current-voltage characteristics. The typical value of zero field $J_{\text {c }}$ ranges from $10^{4}$ to $9 \times 10^{5}$ $\mathrm{A} / \mathrm{cm}^{2}$ at $12 \mathrm{~K}$, depending on the detailed preparation conditions. For comparison, films made by rf magnetron sputtering (also with post-annealing) were also used. ${ }^{9)}$ The rf-sputtered films exhibit a much smoother surface and an epitaxy-quality $c$-axis-oriented structure. The critical current density for these films was very high, typically $5 \times 10^{5} \mathrm{~A} / \mathrm{cm}^{2}$ at $90 \mathrm{~K}$.

Besides the apparent microstructure differences, the temperature-dependent critical current and resistivity for the films used also exhibit a variety of distinct features, as shown in Fig. 1. It is clear that all the films manifested a pronounced dissimilarity in transition temperature, morphology and critical current density under different preparation conditions. As shown in Fig. 1(a), the zero resistance transition temperatures of both the representative dc-sputtered (B, C, D) and rf-sputtered (A) films ranged from $65 \mathrm{~K}$ to $106 \mathrm{~K}$, indicating the relative quality of the films. In comparison, the temperature-dependent critical current densities for films A-D are plotted in Fig. 1(b). It is clear that in addition to the conspicuous differences in the magnitude and slope of $J_{\mathrm{c}}(T)$, the films which had a lower $T_{\text {co }}$ possessed a much higher-value $J_{\mathrm{c}}$ in the low-temperature region (e.g. compare samples $\mathrm{B}$ and $\mathrm{C}$ in Fig. 1(b)). The X-ray diffraction analyses revealed that while all films were composed of both $\mathrm{Tl}_{2} \mathrm{Ca}_{2} \mathrm{Ba}_{2} \mathrm{Cu}_{3} \mathrm{O}_{x}$ and $\mathrm{Tl}_{2} \mathrm{Ca}_{1} \mathrm{Ba}_{2} \mathrm{Cu}_{2} \mathrm{O}_{x}$ phases, there were differences in the degree of preferred orientation. The results suggested that the transport critical current is dominated by the degree of preferred orientation of the 

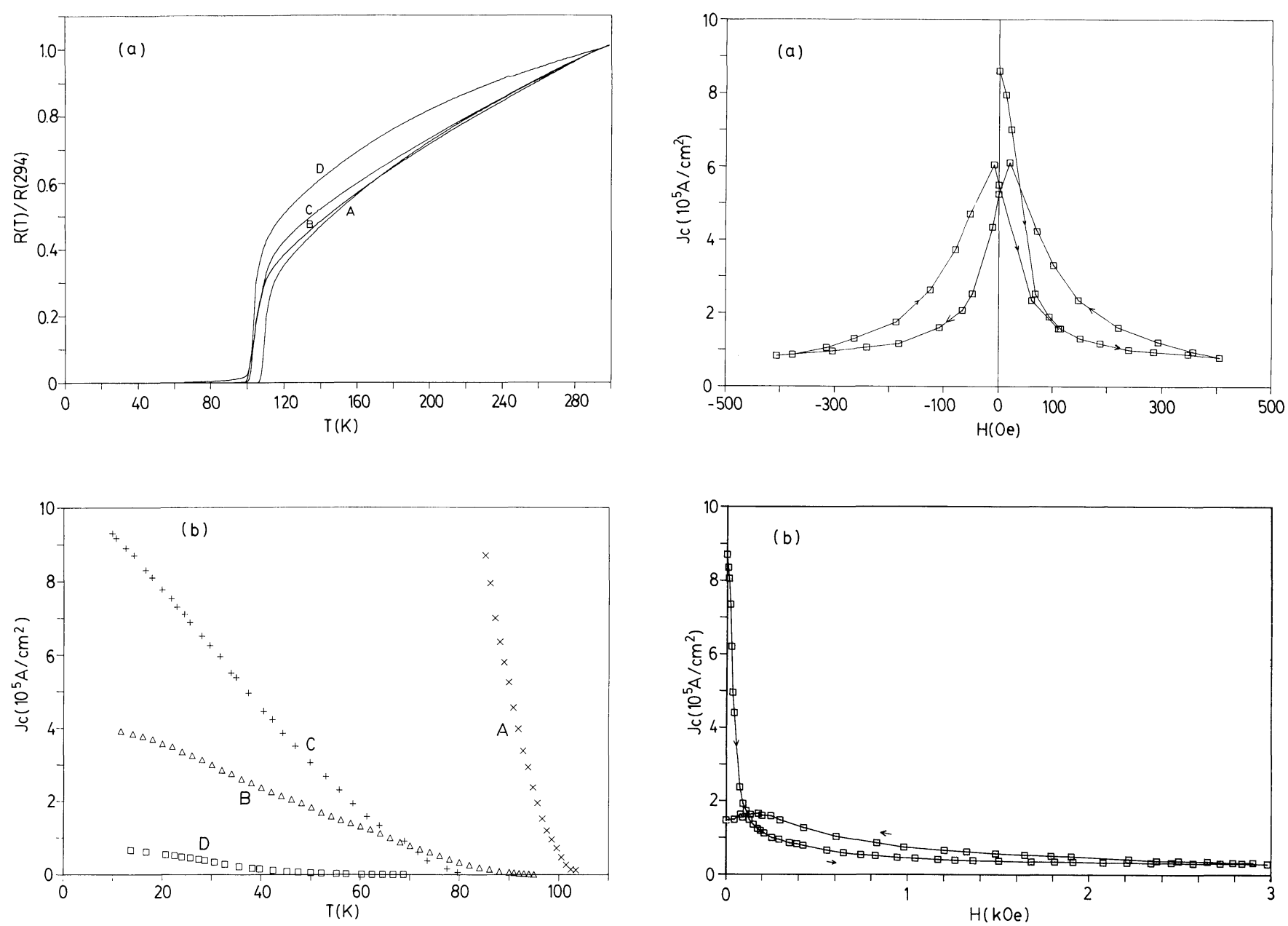

Fig. 1. The temperature dependences of the (a) normalized resistivity and (b) critical current density for the rf (Sample A) and dc (Samples $\mathrm{B}, \mathrm{C}, \mathrm{D})$ sputtered $\mathrm{Tl}-\mathrm{Ca}-\mathrm{Ba}-\mathrm{Cu}-\mathrm{O}$ superconducting thin films.

superconducting grains rather than the relative concentration of both superconducting phases. It is also noted that the differences in transport $J_{\mathrm{c}}$ may also arise from the detailed microstructure which is closely related to the pinning mechanisms.

With the variety of films as described above, we can now proceed to see how $J_{\mathrm{c}}(H)$ will behave in each of these films. Figure 2 illustrates the transport critical current density measured for sample $\mathrm{C}$ as a function of the applied magnetic field after zero-field cooling (ZFC) to $12.5 \mathrm{~K}$. The direction of the field was applied perpendicular to the plane of the film, and the same configuration was maintained for all the other measurements. The transport $J_{\mathrm{c}}$ of the "virgin" curve falls precipitously with increasing applied field, as shown in Figs. 2(a) and 2(b). The results are consistent with the distinct feature of the granular superconducting thin films. ${ }^{1,10)}$ However, as the magnetic field monotonically increases to its maximum value $H_{\mathrm{m}}$, the field-decreasing branches of $J_{\mathrm{c}}$ are apparently enhanced over the virgin curves. In addition, peaks occur before the applied field is reduced to zero with the peak heights depending on the $H_{\mathrm{m}}$ values (cf. Figs. 2(a) and 2(b)). When the field is reduced to zero, the critical current density is much lower than that of the virgin

Fig. 2. Hysteresis of the transport critical current density for Sample $\mathrm{C}$ after zero-field cooling to $12.5 \mathrm{~K}$. The cycle of the applied magnetic field proceeded as indicated by the arrows. The maximum applied field was 405 Oe (a) and 2980 Oe (b).

state. The $J_{\mathrm{c}}(H)$ curve in the negative field region shows a symmetrical image to the positive curve with the symmetry axis located around $H=6$ Oe (see Fig. 2(a)). This value was interpreted as the effect of the self-field. ${ }^{2)}$ Similar behaviors were observed in other Tl-based granular thin films except for sample A, which will be discussed in detail later. As has been mentioned previously, the hysteresis of critical current density in low magnetic field has been conjectured to be a common feature of granularity. Accordingly, the weak-link network (WLN) formed in the disorderly permuted superconducting grains may form some supercurrent loops due to the diamagnetic flux gradients on the increasing field portion to trap some flux when the intergranular $H_{\mathrm{c} 2 \mathrm{j}}$ is exceeded. The establishment of the supercurrent loops exerts strong influence on the phase relations between grains as well as the transport current through the grains..$^{2-5)}$ As the applied field is reduced from $H_{\mathrm{m}}$, the flux gradients and supercurrent loops will be reversed. Thus, if the diamagnetic current acts to depress the intergranular critical current, the reversed paramagnetic current will act contrarily, resulting in an irreversible $J_{\mathrm{c}}(H)$. If this is true, one would expects the transport $J_{\mathrm{c}}$ to be an approximately single-valued function of the applied magnetic 
field for the sample whose intergranular $H_{\mathrm{c} 2 \mathrm{j}}$ is larger than $H_{\mathrm{m}}$. Indeed, the results of reversible transport critical current density were observed for sample $\mathrm{C}$ with applied field smaller than $50 \mathrm{Oe}$ at $12.5 \mathrm{~K}$.

In contrast to the weak-link dominated behavior described above, nonhysteretic $J_{\mathrm{c}}(H)$ was observed for sample A. Figure 3 shows the results measured at 91, 95 and $97 \mathrm{~K}$ with the applied $H_{\mathrm{m}}$ of 1380,858 and 667 Oe, respectively. These results are similar to that observed in $\mathrm{Bi}-\mathrm{Sr}$ $\mathrm{Ca}-\mathrm{Cu}-\mathrm{O} / \mathrm{Ag}$ measured at $77 \mathrm{~K} .^{7)}$ In ref. 7 , the lack of hysteretic $J_{\mathrm{c}}(H)$ was attributed to the absence of both pinning and weak-link structures, leading to a huge thermally activated flux motion which repressed the hysteretic effect. However, since in our case the critical current remained very high even at temperatures close to $T_{\text {co }}$, it seems that the lack of weak links is more active in the observed differences. Combined with the results of sample $\mathrm{C}$ at lower fields, this implies an intergranular $H_{\mathrm{c} 2 \mathrm{j}}$ larger than many hundred oersteds, which is not surprising considering the near-epitaxial quality of the rf sputtered films.

While the hysteretic $J_{\mathrm{c}}(H)$ obtained after zero-field cooling proved to be very sample-dependent and mostly due to the flux trap in the weak-link network structure, similar measurements performed in the field-cooled (FC) state elicited completely different results. Figures 4(a) and 4(b) show the typical $J_{\mathrm{c}}$ curves of samples $\mathrm{C}$ and D as functions of decreasing applied field at 12.5 and $16.5 \mathrm{~K}$ after field-cooling from various magnetic fields. Apparent enhancement of $J_{\mathrm{c}}$ is observed as compared to the ZFC virgin curve which is not shown in Fig. 4. (cf. e.g., Fig. 2). In addition to this enhancement, it is noted that the peak positions of $J_{\mathrm{c}}$ in the FC states have very weak dependence on the initial field-cooled values $\left(H_{\mathrm{FC}}\right)$. This is very different from what was observed in the $\mathrm{Y}-\mathrm{Ba}-$ $\mathrm{Cu}-\mathrm{O}$ system ${ }^{2)}$ where a steep rise in $J_{\mathrm{c}}$ near the applied $H_{\mathrm{FC}}$ was observed. The results, while interesting, are difficult to reconcile due to the sample-dependent characteristics of the complicated connected WLN. A tempting interpretation is that under a FC state with the applied $H_{\mathrm{FC}}$ larger than its intergranular $H_{\mathrm{c} 2 \mathrm{j}}$, the amount of flux

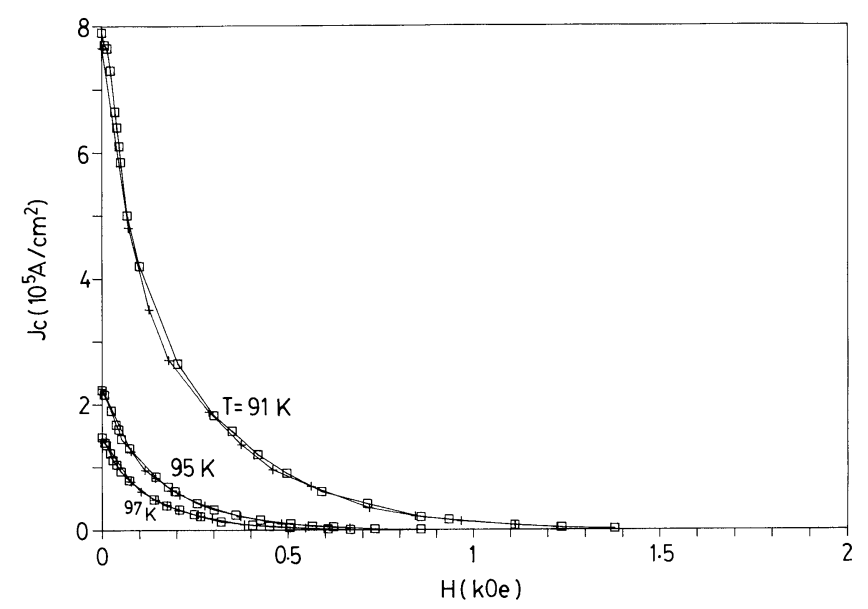

Fig. 3. The field dependences of the critical current density for Sample A after zero-field cooling to 91,95 and $97 \mathrm{~K}$; $\square$, field increase; +, field decrease. trapped in each supercurrent loop has an upper limit regardless of the initial $H_{\mathrm{FC}}$. Since the hysteretic effects are mostly due to the amount of the trapped flux, one would expect the same peak location to occur in the $J_{c}(H)$ curves, as observed in Fig. 4. Another important feature is that in Fig. 4(a), the maximum value of $J_{\mathrm{c}}$ varies with the initial fields while in Fig. 4(b), in addition to the small but evident oscillations in $J_{\mathrm{c}}$, approximately equal peak height is observed. Again, this is believed to be due to the detailed nature of the WLN and the oscillations of $J_{c}$ could result from the additional Josephson phase slip effects between the weakly coupled grains.

As has been demonstrated above, the trapped flux affects $J_{\mathrm{c}}(H)$ significantly and gives rise to many interesting phenomena. Since the structure of the trapped flux lines depends on both the strength of the applied field and the temperature, it would be illuminating to delineate the influence of temperatures on $J_{\mathrm{c}}(H)$. Details of such an experiment are as follows:

1. Magnetic fleld, which was larger than the intergranular $H_{\mathrm{c} 2 \mathrm{j}}$ of the investigated sample, was applied
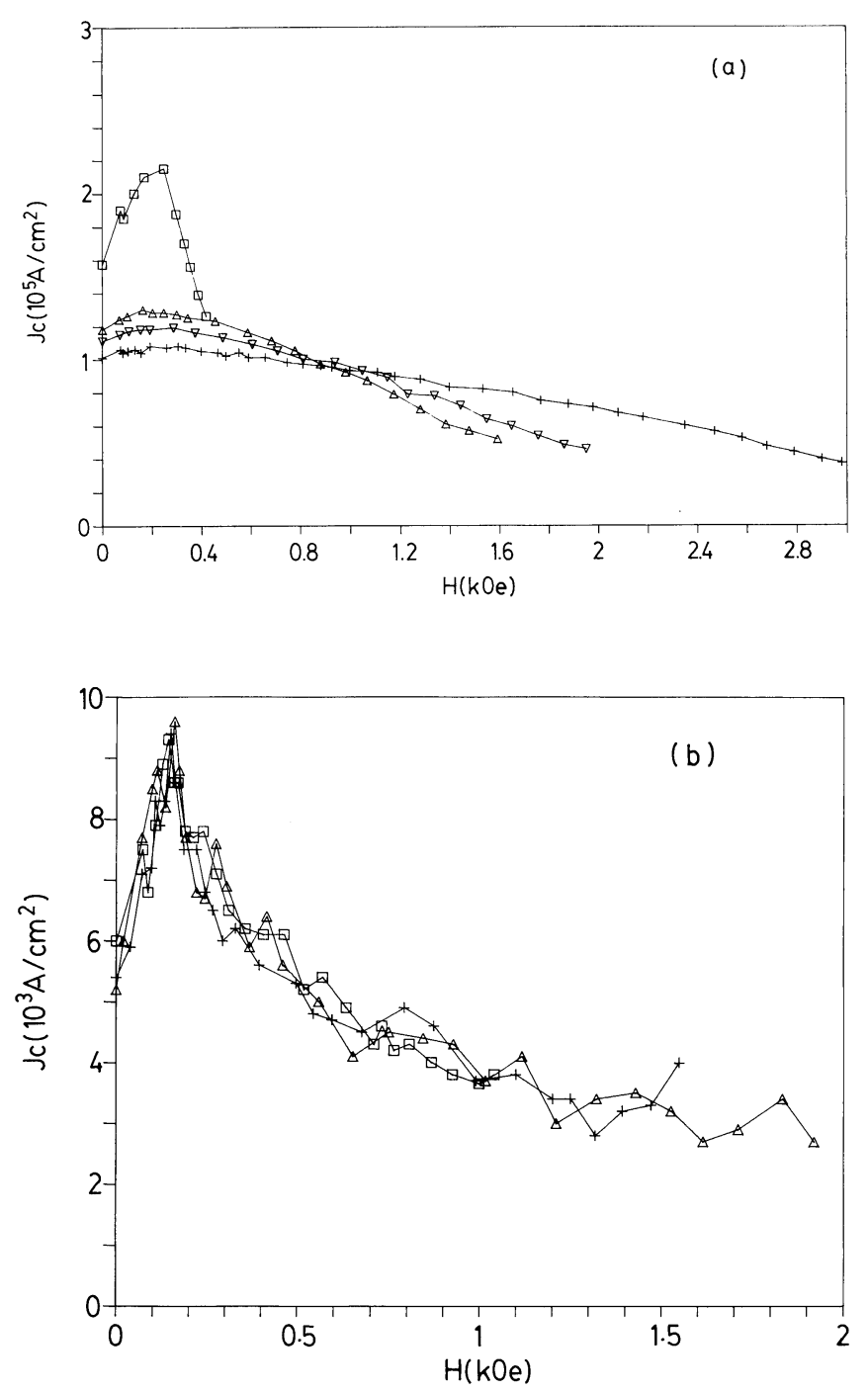

Fig. 4. Critical current density measured in decreasing field after field cooling for (a) Sample C with $H_{\mathrm{FC}}=3000$ Oe $(+) ; 1950$ Oe $(\nabla) ; 1591$ Oe $(\triangle) ; 418 \mathrm{Oe}(\square)$ at $12.5 \mathrm{~K}$. (b) Sample D with $H_{\mathrm{FC}}=1920 \mathrm{Oe}(\triangle)$; $1550 \mathrm{Oe}(+)$; $1040 \mathrm{Oe}(\square)$ at $16.5 \mathrm{~K}$. 
for about $15 \mathrm{~s}$ after zero-field cooling to $14 \mathrm{~K}$.

2. After removing the applied field, the depressed transport critical current density was measured as $J_{\mathrm{c}}(14 \mathrm{~K})$.

3. The temperature was increased to a certain value, named $T_{\text {anneal }}$.

4. The thin film was cooled again to $14 \mathrm{~K}$ and the value of $J_{\mathrm{c}}$ taken, named $J_{\mathrm{c}}^{\prime}(14 \mathrm{~K})$.

5. The difference between $J_{\mathrm{c}}^{\prime}(14 \mathrm{~K})$ and $J_{\mathrm{c}}(14 \mathrm{~K})$ was calculated as $\Delta J_{\mathrm{c}}=J_{\mathrm{c}}^{\prime}(14 \mathrm{~K})-J_{\mathrm{c}}(14 \mathrm{~K})$.

6. All the processes were repeated with a different $T_{\text {anneal }}$. Because of the flux creep effect, the magnetization of a superconductor decays with time logarithmically. ${ }^{11}$ Intuitively, similar time-dependent effects are expected for the trapped-flux-induced $J_{c}$ depression. However, the depressed critical current density of our Tl-based samples remained unchanged for at least 72 hours at temperatures of 12 and $50 \mathrm{~K}$ after removal of the applied field, thus the results presented below should be taken as effects of the temperature rather than of time. In Fig. 5, $\Delta J_{\mathrm{c}}$ versus $T_{\text {anneal }}$ is plotted for sample B as a representative; the dotted line is drawn to guide the eye. Note that there are three distinct regions present in the whole range of temperatures. Below $30 \mathrm{~K}, \Delta J_{\mathrm{c}}$ is essentially independent of $T_{\text {anneal }}$. The plateau between approximately $38 \sim 50 \mathrm{~K}$ manifested the second area where $\Delta J_{\mathrm{c}}$ is no further enhanced after the initial enhancement. While $T_{\text {anneal }}$ is higher than $50 \mathrm{~K}, \Delta J_{\mathrm{c}}$ is linearly increased. Similar $\Delta J_{\mathrm{c}}$ behavior was found in other Tl-based granular thin films with slight differences in "transition temperatures", which divides the characteristic of $\Delta J_{c}$ into the three regions. Two possible explanations of the observed results are proposed. Firstly, the magnetic flux lines which were trapped in the films may escape due to the thermally activated energy induced by the heating process. Alternatively, the equilibrium structure of flux lines

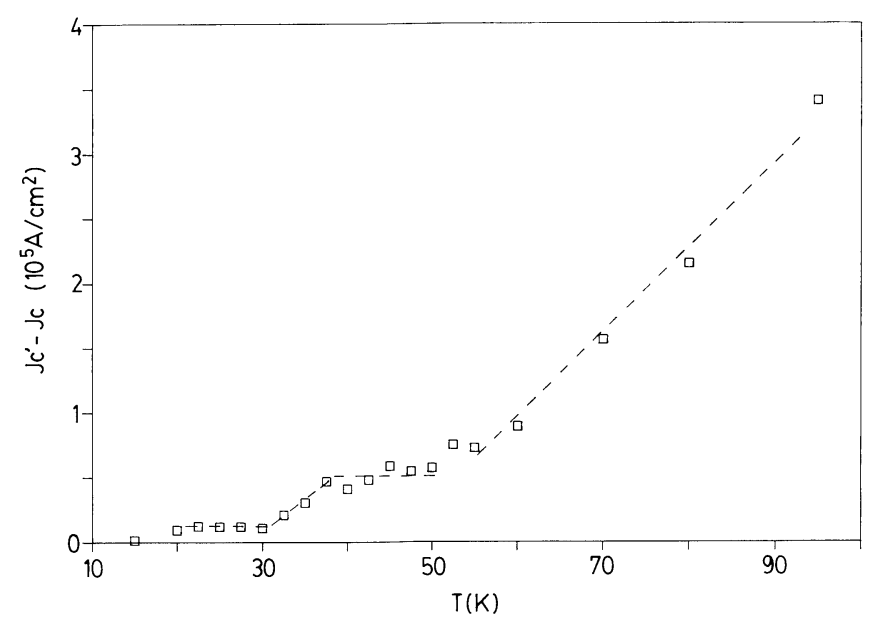

Fig. 5. $\Delta J_{\mathrm{c}}$ versus the annealing temperature for Sample B. in the Tl-based superconducting material may exhibit different phases in the temperature range studied. This would determine the amount of flux which remained trapped at $T_{\text {anncal }}$, and hence exert various influences on the depressed $J_{\text {c }}$. Considering the time-independence effect, the latter seems a more plausible explanation to give a consistent picture. In light of these factors, Fig. 5 reflects that in the temperature range from $14 \mathrm{~K}$ to above $90 \mathrm{~K}$, there are three equilibria or dynamic states for the flux lines in $\mathrm{Tl}-\mathrm{Ca}-\mathrm{Ba}-\mathrm{Cu}-\mathrm{O}$ superconducting thin films. This may further correlate to the Abrikosov, vortex glass, and flux-line-lattice melting states suggested by many independent studies of the whole family of oxide superconductors. ${ }^{12,13)}$

In summary, we have investigated the behavior of the transport critical current density of $\mathrm{Tl}-\mathrm{Ca}-\mathrm{Ba}-\mathrm{Cu}-\mathrm{O}$ superconducting thin films in a cycling magnetic field. Hysteresis of the transport $J_{c}$ was observed after zerofield cooling and features similar to those found in the polycrystalline $\mathrm{Y}-\mathrm{Ba}-\mathrm{Cu}-\mathrm{O}$ and granular $\mathrm{Nb}, \mathrm{Al}$ thin films were manifested. The flux trapped by the supercurrent loops in the WLN when the applied field exceeds the intergranular $H_{\mathrm{c} 2 \mathrm{j}}$ of the films is considered to be responsible for the irreversibility of the critical current density. A novel result of the transport $J_{c}$ of field-cooled samples has been discovered. Furthermore, the effects of temperature on both the trapped flux and the transport $J_{\mathrm{c}}$ suggest the existence of various equilibria or dynamic states of the trapped flux in the Tl-based superconducting thin films.

The authors wish to thank Mr. C. Wang and Mr. M. L. Chu for experimental assistance and helpful discussions. This work at National Chiao Tung Univ. was supported by National Science Council of R.O.C. under Contract NSC79-0208-M009-38.

\section{References}

1) M. Tinkham and C. J. Lobb: Solid State Phys. 42 (1989) 91.

2) M. E. McHenry, M. P. Maley and J. O. Willis: Phys. Rev. B 40 (1989) 2666.

3) Y. J. Qian, Z. M. Tang, K. Y. Chen, B. Zhou, J. W. Qiu, B. C. Miao and Y. M. Cai: Phys. Rev. B 39 (1989) 4701.

4) J. E. Evetts and B. A. Glowacki: Cryogenics 28 (1988) 641.

5) K. Watanabe, K. Noto, H. Morita, H. Fujimori, K. Mizuno, T. Aomine, B. Ni, T. Matsushita, K. Yamafuji and Y. Muto: Cryogenics 29 (1989) 263.

6) T. Aomine and A. Yonekura: Phys. Lett. A 114 (1986) 16.

7) K. Heine, J. Tenbrink and M. Thöner: Appl. Phys. Lett. 55 (1989) 2441.

8) H. L. Chang, C. Wang, M. L. Chu, T. M. Uen and Y. S. Gou: Jpn. J. Appl. Phys. 28 (1989) L631.

9) S. H. Liou: Proc. Mater. Res. Soc. 169 (1990).

10) R. L. Peterson and J. W. Ekin: Phys. Rev. B 37 (1988) 9848.

11) M. Tinkham: Introduction to Superconductivity (McGraw-Hill, New York, 1975) Chap. 5, p. 175.

12) P. L. Gammel, L. F. Schneemeyer, J. V. Waszczak and D. J. Bishop: Phys. Rev. Lett. 61 (1988) 1666.

13) R. J. Soulen Jr. and S. A. Wolf: Physica C 166 (1990) 95. 